\title{
Quasilinear theory of quantum Fermi liquid
}

\author{
Nodar L. Tsintsadze and Davit M. Alkhanishvili \\ Faculty of Exact and Natural Sciences and E. Andronikashvili Institute of Physics, Javakhishvili Tbilisi State University \\ Tbilisi 0128, Georgia \\ E-mail:nltsin@yahoo.com
}

Received May 1, 2016, published online October 24, 2016

\begin{abstract}
Quasilinear theory of a weakly turbulent quantum Fermi liquid is presented. Landau's linear theory of Fermi liquids is generalized by consideration of weak nonlinear regime. A newly derived kinetic equation of the Fermi particles is used to derive a slowly varying distribution function $f_{0}$, which satisfies the diffusion equation. It is shown that the magnitude of the diffusion coefficient $D$ depends on weak interactions between atoms and the de Broglie waves diffraction.
\end{abstract}

PACS: 67.30.-n ${ }^{3} \mathrm{He}$;

71.10.Ay Fermi-liquid theory and other phenomenological models;

74.20.Mn Nonconventional mechanisms.

Keywords: Fermi liquid, quasiparticles, quasilinear theory.

In 1961 quasilinear theory for plasma particles was created by A.A. Vedenov et al. [1] and W. Dramond and D. Pines [2]. The quasilinear theory describes the dynamics of the interaction between the resonance particles and the waves. The theory is able to treat such processes when the energy of the medium oscillations is appreciably less than the total internal energy of the particles, but is, at the same time, much greater than the noise energy of the sound waves.

The quasilinear theory lies in the division of the particle distribution function into two parts: a rapidly oscillating part and a slowly varying part, and also in calculating the influence of the mean square of the oscillating part of the number density of particles on the slowly varying part. It is found that the behavior of the slow part of the distribution function can be described by a diffusion equation in the momentum space, but the rate of damping or growth of the rapid oscillations is given by the linear theory equations in which the slowly varying part of the distribution function varies slowly with time.

We have applied quasilinear theory to the Fermi liquid. It is well known that at temperatures $1-2 \mathrm{~K}$ only two quantum liquids exist in nature, the isotopes of helium ${ }^{3} \mathrm{He}$ and ${ }^{4} \mathrm{He}$, and all other substances solidify. The peculiarly weak interaction between the helium atoms is the reason for helium to remain liquid. Based on this fact, namely that in ${ }^{3} \mathrm{He}$ the weak interactions take place between atoms at sufficiently low temperatures, Landau has created the theory of Fermi liquid [3]. In which he took into account only the weakly excited energy levels of the liquid, lying fairly close to the ground state. Landau assumed that any weakly excited state of a macroscopic body can be represented as an assembly of separate elementary excitations (quasiparticles). Moreover, the elementary excitations are represented as the collective motion of atoms in liquid and it can not be identified with individual atoms. Therefore, an important characteristic of the energy spectrum is the establishment of the dispersion relation $\varepsilon(p)$ for elementary excitations. Landau has then shown that the undamped zero sound can exist in an almost ideal Fermi gas, which was confirmed in experiment by W.R. Abel et al. [4]. Pomeranchuk has shown that Landau's liquid can be unstable [5]. Landau's theory of Fermi liquids was generalized by incorporating the de Broglie waves diffraction [6].

In this article, we describe the quasilinear theory of Fermi liquids by taking into account the de Broglie wave diffraction and derive the corresponding diffusion equation in momentum space written for equilibrium distribution function. To achieve this we employ a novel quantum kinetic equation derived in Ref. 6, where use was made of the quasiclassical function $\psi=A \exp (i S / \hbar)$. The difference between the Landau kinetic equation and ours is that in our equation an additional term, namely the Madelung term is incorporated due to the diffraction of de Broglie waves.

Nonequilibrium states of a Fermi quantum liquid are described by the one particle distribution function $f(r, p, t)$, which satisfies the quantum Boltzmann equation [6]:

$$
\frac{\partial f}{\partial t}+(\mathbf{v} \cdot \nabla) f-\nabla \varepsilon \cdot \frac{\partial f}{\partial \mathbf{p}}+\frac{\hbar^{2}}{2 m} \nabla \frac{1}{\sqrt{n}} \Delta \sqrt{n} \frac{\partial f}{\partial \mathbf{p}}=C(f),
$$


$\varepsilon$ is the energy of the quasiparticle, $m$ and $n$ are mass and density of particles, respectively, and $C(f)$ is the collision integral, which describes the variation of the distribution function due to particle collisions.

Note that when the spin of the particles is taken into account, the distribution function is an operator with respect to the spin variables $\sigma$. The quasiparticles in a Fermi liquid have spin $1 / 2$. However, there is a wide range of problems in which it is sufficient to consider a distribution independent of spin variables, so that $f$ becomes the ordinary quasiclassical distribution function $f(\mathbf{r}, \mathbf{p}, t)$. We recall here that the condition for quasiclassical motion is that the de Broglie wavelength $\lambda_{d}=\hbar / p_{F}$ ( $p_{F}$ is Fermi momentum) of the particle must be very small compared with the characteristic length $L$, over which $f(\mathbf{r}, \mathbf{p}, t)$ varies considerably. Following the Landau's theory, hereafter, we consider Fermi liquid as a spinless, and the energy $\varepsilon$ of quasiparticle is a functional of the distribution function; a variation of distribution function

$$
f(\mathbf{r}, \mathbf{p}, t)=f_{0}(\mathbf{p})+\delta f(\mathbf{r}, \mathbf{p}, t)
$$

produces a variation of energy given by

$$
\delta \varepsilon=\int \phi\left(\mathbf{p}, \mathbf{p}^{\prime}\right) \delta f\left(\mathbf{r}, \mathbf{p}^{\prime}, t\right) \frac{2 d^{3} \mathbf{p}^{\prime}}{(2 \pi \hbar)^{3}}
$$

where the factor 2 appears due to spin, $f_{0}(\mathbf{p})$ and $\phi\left(\mathbf{p}, \mathbf{p}^{\prime}\right)$ are the equilibrium distribution function and the quasiparticle interaction function, respectively; in a Fermi gas $\phi=0$. Thus the distribution function (2) refers to the energy of quasiparticle

$$
\varepsilon=\varepsilon_{0}(\mathbf{p})+\delta \varepsilon(t, \mathbf{r}, \mathbf{p})
$$

where $\varepsilon_{0}(\mathbf{p})$ is the energy corresponding to the equilibrium state.

Near the surface of the Fermi sphere the variation of distribution function $\delta f\left(\mathbf{r}, \mathbf{p}^{\prime}, t\right)$ is appreciably different from zero, i.e., the magnitude $\mathbf{p}^{\prime}=p=p_{F}$. The same is true for the function $\phi\left(\mathbf{p}, \mathbf{p}^{\prime}\right)$. So that both depend only on directions of the vectors $\mathbf{p}$ and $\mathbf{p}^{\prime}$. Hence, the quasiparticle interaction function $\phi$ and $\delta f$ can be expressed at the Fermi surface as

$$
\begin{gathered}
\phi\left(\mathbf{p}, \mathbf{p}^{\prime}\right)=\frac{\pi^{2} \hbar^{3}}{m^{*} p_{F}} Q(\theta), \\
\delta f=\delta\left(\varepsilon-\varepsilon_{F}\right) F\left(\mathbf{n}^{\prime}, \mathbf{r}, t\right)
\end{gathered}
$$

where $m^{*}=p_{F} / v_{F}$ is the effective mass of quasiparticle, $\mathbf{n}^{\prime}$ is the unit vector in the direction of $\mathbf{p}^{\prime}$, and $Q(\theta)$ is the function of the angle $\theta$ between $\mathbf{p}$ and $\mathbf{p}^{\prime}$.

We now employ Eq. (1) to study the propagation of small perturbations in the Fermi quantum liquid. We substitute the Eqs. (2) and (4) into Eq. (1) and linearize it with respect to the perturbation $\delta f$ to obtain $\frac{\partial \delta f}{\partial t}+(\mathbf{v} \cdot \nabla) \delta f-\nabla \delta \varepsilon \cdot \frac{\partial f_{0}}{\partial \mathbf{p}}++\frac{\hbar^{2}}{4 m} \nabla \cdot \Delta \frac{\delta n}{n_{0}} \frac{\partial f_{0}}{\partial \mathbf{p}}=C(\delta f)$.

We look for wave solutions in space and time for $F\left(\mathbf{n}^{\prime}, \mathbf{r}, t\right)$, assuming that it is proportional to exp $[i(\mathbf{k} \cdot \mathbf{r}-\omega t)]$.

Taking into account Eqs. (3)-(6), $\nabla \varepsilon$ can be written as

$$
\nabla \delta \varepsilon=\int Q\left(\theta^{\prime}\right) \nabla F\left(\mathbf{n}^{\prime}, \mathbf{r}, t\right) \frac{d \Omega^{\prime}}{2}=i \mathbf{k} \int Q\left(\theta^{\prime}\right) F\left(\mathbf{n}^{\prime}\right) \frac{d \Omega^{\prime}}{2}
$$

where $d \Omega^{\prime}=\sin \theta^{\prime} d \theta^{\prime}$.

If we assume that $Q\left(\theta^{\prime}\right)$ is constant, i.e. $Q\left(\theta^{\prime}\right)=Q_{0}$, then

$$
\nabla \delta \varepsilon=i \mathbf{k} Q_{0} \mathrm{e}^{i(\mathbf{k} \cdot \mathbf{r}-\omega t)} \int F\left(\mathbf{n}^{\prime}\right) \frac{d \Omega^{\prime}}{2} .
$$

For density perturbation $\delta n$ we get

$$
\delta n=\int \delta f\left(\mathbf{r}, \mathbf{p}^{\prime}, t\right) \frac{2 d^{3} \mathbf{p}^{\prime}}{(2 \pi \hbar)^{3}}=\frac{4 \pi m^{*} p_{F}}{(2 \pi \hbar)^{3}} \int F\left(\mathbf{n}^{\prime}\right) d \Omega^{\prime} .
$$

Since $n_{0}=8 \pi p_{F} / 3(2 \pi \hbar)^{3}$,

$$
\frac{\delta n}{n_{0}}=\frac{3 m^{*}}{p_{F}^{2}} \mathrm{e}^{i(\mathbf{k} \cdot \mathbf{r}-\omega t)} \int F\left(\mathbf{n}^{\prime}\right) \frac{d \Omega^{\prime}}{2}
$$

and

$$
\nabla \delta \varepsilon=i \mathbf{k} Q_{0} \frac{p_{F}^{2}}{3 m^{*}} \frac{\delta n}{n_{0}} .
$$

To see what impact does quasiparticle collisions have on Fermi liquid, for a rough estimate of collision integral, we can put

$$
C(\delta f)=\frac{f_{0}-f}{\tau}=-\frac{\delta f}{\tau}=-v \delta f
$$

where $\tau=v^{-1}$ is the mean free time.

We substitute Eqs. (12) and (13) into Eq. (7) and finally for perturbation $\delta f$ we get

$$
\delta f=-\left(\frac{p_{F}^{2} Q_{0}}{3 m^{*}} \frac{1}{n_{0}}+\frac{\hbar^{2} k^{2}}{4 m^{*}} \frac{1}{n_{0}}\right) \frac{\delta n}{\omega+i v-\mathbf{k} \cdot \mathbf{v}} \mathbf{k} \cdot \frac{\partial f_{0}}{\partial \mathbf{p}} .
$$

The main criteria for using linear approximation is

$$
|\delta f| \ll f_{0} .
$$

In other words this means, that oscillation energy due to perturbation must be less than Fermi liquid internal energy. For degenerate Fermi liquid this condition has form

$$
\frac{W}{N \varepsilon_{F}} \ll 1
$$

where $N \varepsilon_{F}$ is an internal energy of the Fermi liquid and $W$ is the oscillation energy due to perturbation.

Condition (16) holds true for the most mass of the liquid, but there exists some number of particles for which this condition is not maintained. Those are the particles, 
which move in phase with the sound waves and due to this they become involved in Landau damping. In this process the distribution function changes. This change has a nonlinear character and because of this, its behavior greatly depends on spectral composition of the sound wave. In linear theory influence of oscillations on distribution function is neglected. Contrary to this, in quasilinear theory waves are still considered linear, but distribution function change due to oscillations is not neglected.

To study Fermi liquid in quasilinear theory we consider perturbations, which are ensemble of waves and their wave vectors are located inside narrow $\Delta k$ interval around some arbitrary $k_{0}$ wave vector. Because wave vector interval is narrow, small amount of quasiparticles will be involved in the Landau damping and as a result their distribution function will change significantly.

In order to derive the equations in the quasilinear approximation, we split up the distribution function $f(t, \mathbf{p})$ into a large slowly and a small fast developing part:

$$
\begin{gathered}
f=f_{0}(t, \mathbf{p})+\delta f(t, \mathbf{p})=f_{0}(t, \mathbf{p})+\sum_{k} \delta f_{k} \mathrm{e}^{i(\mathbf{k} \cdot \mathbf{r}-\omega t)}, \\
\nabla \delta \varepsilon=\sum_{k} i \mathbf{k} \frac{p_{F}^{2} Q_{0}}{3 m} \frac{\delta n_{k}}{n_{0}} \mathrm{e}^{i(\mathbf{k} \cdot \mathbf{r}-\omega t)},
\end{gathered}
$$

where perturbation $\delta f_{k}$ is defined in Eq. (14).

Second term in the Eq. (17) is a rapidly oscillating term and so it vanishes after a time averaging over fast oscillations. First term $f_{0}(t, \mathbf{p})$ represents slowly varying averaged part of the distribution function.

Now we substitute Eqs. (17) and (18) into Eq. (1) representing quantum-kinetic equation and take a time average over the fast oscillations. The time interval $\tau_{0}$ must satisfy the conditixon

$$
\frac{2 \pi}{\omega} \ll \tau_{0} .
$$

Here $\tau_{0}$ is the time during which the oscillations influence the equilibrium quantum liquid state. Then we have

$$
\langle f(\mathbf{r}, \mathbf{p}, t)\rangle=f_{0}(\mathbf{p}, t)=\frac{1}{\tau_{0}} \int_{0}^{\tau_{0}} f d t .
$$

Due to averaging Eq. (1), we obtain for the slow part of the distribution function:

$$
\frac{\partial f_{0}}{\partial t}-\left\langle\nabla \delta \varepsilon \cdot \frac{\partial \delta f}{\partial \mathbf{p}}\right\rangle+\left\langle\frac{\hbar^{2}}{4 m} \nabla \cdot \Delta \frac{\delta n}{n_{0}} \frac{\partial \delta f}{\partial \mathbf{p}}\right\rangle=0
$$

As a result, after taking an average of Eq. (21), we obtained quasilinear equation for the slow part of the distribution function in momentum space, which has the form

$$
\frac{\partial f_{0}}{\partial t}=\frac{\partial}{\partial p_{i}} D_{i j} \frac{\partial f_{0}}{\partial p_{j}}
$$

where $D_{i j}$ is the tensor of diffusion coefficients

$$
D_{i j}=\sum_{k} k_{i} k_{j}\left(\frac{p_{F}^{2} Q_{0}}{3 m}+\frac{\hbar^{2} k^{2}}{4 m}\right)^{2}\left|\frac{\delta n_{k}}{n_{0}}\right|^{2} \frac{2 v}{(\omega-\mathbf{k} \cdot \mathbf{v})^{2}+v^{2}} .
$$

Theory that is established on this equation is called quasilinear theory. The process absorption can be described with quasilinear theory, which, as we said above, takes into account changing of equilibrium distribution function due to oscillations caused by sound waves. It is apparent, that oscillations must affect distribution function in such way to decrease the absorption of waves (decrease interaction with particles). According to this, by increasing wave amplitude it absorption must decrease and in Fermi liquid, where we neglect collisions, can be established such distribution, where absorption does not happen. From linear theory we know that wave absorption is determined with the value of $\partial f_{0} / \partial p$ (due to Landau damping). Hence, described situation is possible, if due to impact of the wave on the Fermi liquid plateau (area where $\partial f_{0} / \partial p=0$ ) is created on the equilibrium distribution function and at this moment wave is still not absorbed.

When we consider the collision processes, plateau can not be created on the distribution function, because particle collisions tend to establish equilibrium state in the system. We can clearly observe this from the Eq. (14), when $\omega=\mathbf{k} \cdot \mathbf{v}$ (particles which move in phase with the waves are involved in the plateau formation). We get

$$
-i v \delta f=\left(\frac{p_{F}^{2} Q_{0}}{3 m}+\frac{\hbar^{2} k^{2}}{4 m}\right) \mathbf{k} \cdot \frac{\partial f_{0}}{\partial \mathbf{p}} \frac{\delta n}{n_{0}} .
$$

It is clear that $\partial f_{0} / \partial p \neq 0$, so plateau can not formed on the distribution function.

In the absence of interaction between quasiparticles, i.e., $Q_{0}=0$, we see that

$$
\frac{\partial f_{0}}{\partial t}=\frac{\partial}{\partial p_{i}} \sum_{k} k_{i} k_{j}\left(\frac{\hbar^{2} k^{2}}{4 m}\right)^{2}\left|\frac{\delta n_{k}}{n_{0}}\right|^{2} \frac{2 v}{(\omega-\mathbf{k} \cdot \mathbf{v})^{2}+v^{2}} \frac{\partial f_{0}}{\partial p_{j}}
$$

From this we can deduce, that de Broglie wave diffraction causes diffusion of distribution function in the momentum space, when interaction between quasiparticles is absent.

To illustrate the solution of Eq. (22) for simple cases, we assume that diffusion coefficient (23) does not depend on velocity of the quasiparticles and propagating waves wave vectors are parallel to each other. This is possible if $\omega \approx \mathbf{k} \cdot \mathbf{v}$ or $(\omega-\mathbf{k} \cdot \mathbf{v})^{2} \ll v^{2}$. In this case

$$
\begin{gathered}
\frac{\partial f_{0}}{\partial t}=D \frac{\partial^{2} f_{0}}{\partial \mathbf{p}^{2}}, \\
D=\sum_{k} k^{2}\left(\frac{p_{F}^{2} Q_{0}}{3 m}+\frac{\hbar^{2} k^{2}}{4 m}\right)^{2}\left|\frac{\delta n_{k}}{n_{0}}\right|^{2} \frac{2}{v} .
\end{gathered}
$$


The solution of Eq. (26) has the form

$$
f_{0}(t, \mathbf{p})=\frac{1}{8(\pi D t)^{3 / 2}} \int F\left(\mathbf{p}^{\prime}\right) \exp \left[-\frac{\left(\mathbf{p}-\mathbf{p}^{\prime}\right)^{2}}{4 D t}\right] d^{3} p^{\prime}
$$

where $F\left(\mathbf{p}^{\prime}\right)$ is the initial distribution function, i.e., $F(\mathbf{p})=f_{0}(0, \mathbf{p})$.

In our case $F\left(\mathbf{p}^{\prime}\right)$ is distribution function which describes degenerate Fermi liquid at $T=0 \mathrm{~K}$ temperature. Mathematically it is Heaviside step function. So

$$
F(p)= \begin{cases}1 & p \leq p_{F}, \\ 0 & p>p_{F} .\end{cases}
$$

Solution of Eq. (26) in 1D is

$$
f_{0}\left(t, p_{X}\right)=\frac{1}{2}\left[\operatorname{erf}\left(\frac{p_{X}+p_{F}}{2 \sqrt{D t}}\right)+\operatorname{erf}\left(\frac{p_{F}-p_{X}}{2 \sqrt{D t}}\right)\right]
$$

where erf $(x)$ is an error function, which is defined as

$$
\operatorname{erf}(x)=\frac{2}{\sqrt{\pi}} \int_{0}^{x} \mathrm{e}^{-t^{2}} d t
$$

Corresponding plot of Eq. (30) for various moments of time illustrate Fig. 1.

From this plot we can deduce that diffusion of distribution function in momentum space causes violation of the Fermi surface.

Since the diffusion is happening in the momentum space, density of the particles must be constant and must equal to density of the degenerate Fermi liquid. We can check this by integrating Eq. (28).

$n=\frac{2}{(2 \pi \hbar)^{3}} \frac{1}{8(\pi D t)^{3 / 2}} \iint F\left(\mathbf{p}^{\prime}\right) \exp \left[-\frac{\left(\mathbf{p}-\mathbf{p}^{\prime}\right)^{2}}{4 D t}\right] d^{3} p^{\prime} d^{3} p$,

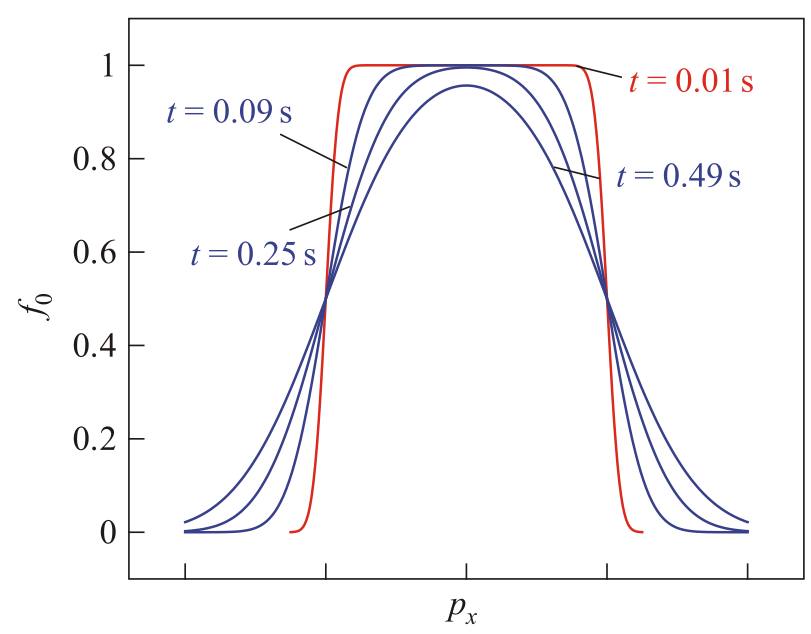

Fig. 1. (Color online) Plot of the distribution function, represented by Eq. (30) for various moments of time.

$$
n=\frac{p_{F}^{3}}{3 \pi^{2} \hbar^{3}}
$$

As we expected solution (28) gives physical results and thus our theory is correct.

In the similar way we calculated mean energy using the distribution function (28)

$$
\begin{gathered}
E=\frac{2}{(2 \pi \hbar)^{3}} \frac{1}{8(\pi D t)^{3 / 2}} \int \frac{p^{2}}{2 m} \int F\left(\mathbf{p}^{\prime}\right) \exp \left[-\frac{\left(\mathbf{p}-\mathbf{p}^{\prime}\right)^{2}}{4 D t}\right] d^{3} p^{\prime} d^{3} p \\
E=\frac{3 p_{F}^{2} n}{10 m}+\frac{6 D t n}{2 m} .
\end{gathered}
$$

First term of this expression is the mean energy in absence of diffusion and corresponds to energy of degenerate Fermi liquid. Second term arises due to diffusion and increases linearly with time.

To summarize, we have developed quasilinear theory of Fermi liquids by taking into account the diffraction of the de Broglie waves. To this end we used the quantum kinetic equation derived by N.L. Tsintsadze and L.N. Tsintsadze in recent paper [6]. It should be noted that our kinetic equation is considerably richer than the Landau's kinetic equation. There is an additional physical feature included here, namely the Madelung term is incorporated due to the diffraction of the de Broglie waves. This term is responsible for the diffusion in the momentum space even in an ideal Fermi gas. Quasilinear theory can be also applied to electrons in most metals. The theory has found further application in nuclear an neutron star matter, superfluid ${ }^{3} \mathrm{He}$ and contemporary problems in superconductivity.

\section{Acknowledgments}

N.L.T. and D.M. Alkhanishvili would like to acknowledge the partial support of GNSF Grant Project N FR/101/6-140/13.

1. A.A. Vedenov, E.P. Velikov, and R.Z. Sagdeev, Usp. Fiz. Nauk 23, 701 (1961).

2. W. Drammond and D. Pines, Proceeding of the Int. Conference on Plasma Physics and Controlled Thermonuclear Fusion, Salzburg (1961), Paper No. 134.

3. L.D. Landau, Zh. Eksp. Teor. Fiz. 30, 1058 (1956); ibid. 32, 59 (1957); ibid. 35, 97 (1958).

4. W.R. Abel, A.C. Anderson, and J.C. Wheatley, Phys. Rev. Lett. 17, 74 (1966).

5. I.Ia. Pomeranchuk, Sov. Phys. JETP 35, 524 (1958).

6. N.L. Tsintsadze and L.N. Tsintsadze, Europhys. Lett. 88, 35001 (2009); From Leonardo to ITER: Nonlinear and Coherence Aspects, Jan Weiland (ed.), AIP Proc. No. CP1177 AIP, New York (2009).

7. N.L. Tsintsadze and L.N. Tsintsadze, Fiz. Nizk. Temp. 37, 982 (2011) [Low Temp. Phys. 37, 782 (2011)]. 